Gynäkologe 2006 · 39:277

DOI 10.1007/s00129-006-1828-z

Online publiziert: 13. April 2006

๑) Springer Medizin Verlag 2006

K. Vetter

Klinik für Geburtsmedizin, Vivantes Klinikum Neukölln, Berlin

\title{
Update Pränatal- und Geburtsmedizin
}

Die Schwangerschaft ist in der Wahrnehmung der Bevölkerung ein quasi gelöstes Problem - grundsätzliche Veränderungen sind scheinbar dank der stabilen Kanalisierung von Ansprüchen und Leistungen durch die Mutterschafts-Richtlinien nicht $z u$ berïcksichtigen. Einen Hinweis darauf, dass dem gar nicht so ist, soll dieses Heft geben. Es behandelt ausschnitthaft Veränderungen in Pränatal- und Geburtsmedizin, die sehr wohl nicht nur unser Denken über die Schwangerschaft, sondern auch unser Handeln während der Schwangerschaft beeinflussen und schließlich einen Teil zur Morgenröte beitragen, die auf der Hoffnung fußt, prospektives Handeln im Sinn der Eltern und der entstehenden neuen Generation sei durch neue Erkenntnisse möglich.

\section{( ) Veränderungen in Pränatal- und Geburtsmedizin beeinflussen unser Denken und Handeln während der Schwangerschaft}

Zunächst stellt sich die Frage, ob der transportable papierne Mutterpass dazu geeignet ist, die aktuell relevanten Informationen ohne Verluste - aufgrund von Doppel-oder Extradokumentation - zu transferieren. Elektronische Möglichkeiten werden derzeit ausgelotet. Hier wird von der Gruppe um Roland Zimmermann ein in Zürich schon praktiziertes Konzept der Datenübermittlung für Schwangere und Ärzte dargestellt, das dazu geeignet ist, die entsprechenden Diskussionen zu fokussieren.

Pränataldiagnostik im weiteren Sinn bietet heute für die Schwangerschafts-, aber auch für die Geburtsplanung Möglichkeiten, die vor einigen Jahren noch nicht denkbar waren. Individuelle Risiken für das Kind, für die Mutter und für die Schwan- gerschaft lassen sich nicht nur in Bezug auf die Anlage der Schwangerschaft eruieren. Joachim Hackelöer zeigt auf, dass ebenso Geburtsplanung und Durchführung der Geburt nicht unerheblich von den Erkenntnissen der Bild gebenden Diagnostik profitieren können.

Neben der Unterversorgung des Ungeborenen spielen Frühgeburten medizinisch und gesellschaftlich die größte Rolle unter den beeinflussbaren Problemen. Deshalb werden diesem Thema zwei Beiträge gewidmet.

Zunächst fasst Irene Hösli die Möglichkeiten der Verhinderung von Frühgeburten zusammen. Dies tut sie nicht nur unter dem Gesichtspunkt der Hoffnung, sondern der Relevanz vorgeschlagener und geübter Methoden.

Welche Möglichkeiten es gibt, mit einer drohenden Frühgeburt umzugehen, stellt Ernst Beinder dar. Er hat sich der Aufgabe gemacht, vorhandene Konzepte unter unterschiedlichen Gesichtspunkten objektiv zu beleuchten, um dem Leser die Basis für seine informierte Entscheidung $z u$ schaffen, die er in der klinischen Situation treffen muss.

In diesem Sinne sei den Lesern eine informative Lektüre gewünscht!

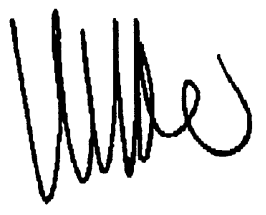

Prof. Dr. K. Vetter

\section{Korrespondierender Autor}

\section{Prof. Dr. K. Vetter}

Klinik für Geburtsmedizin

Vivantes Klinikum Neukölln

Rudower Straße 48, 12351 Berlin

klaus.vetter@vivantes.de 